Revue internationale de l'économie sociale

Recma

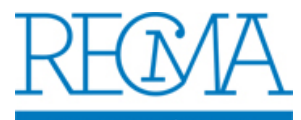

\title{
Regards sur les monnaies sociales et complémentaires
}

\section{Jérôme Blanc}

Numéro 324, avril 2012

Monnaies sociales et complémentaires

Community and Complementary Currencies

URI : https://id.erudit.org/iderudit/1017774ar

DOI : https://doi.org/10.7202/1017774ar

Aller au sommaire du numéro

\section{Éditeur(s)}

Association Recma

ISSN

1626-1682 (imprimé)

2261-2599 (numérique)

Découvrir la revue

\section{Citer ce document}

Blanc, J. (2012). Regards sur les monnaies sociales et complémentaires. Revue internationale de l'économie sociale, (324), 18-20.

https://doi.org/10.7202/1017774ar d'utilisation que vous pouvez consulter en ligne.

https://apropos.erudit.org/fr/usagers/politique-dutilisation/ 


\title{
REGARDS SUR LES MONNAIES SOCIALES ET COMPLÉMENTAIRES
}

\author{
par Jérôme Blanc*
}

\footnotetext{
* Maître de conférences HDR, université Lumière-Lyon 2 , et chercheur au laboratoire Triangle, UMR 5206. Mél.: jerome.blanc@ univ-lyon2.fr.
}

- e dossier qui suit est constitué de quatre textes dont des versions préliminaires ont été présentées lors du colloque international, trilingue et pluridisciplinaire "Trente années de monnaies sociales et complémentaires, et après? ", qui s'est tenu à l'Ecole normale supérieure (ENS) de Lyon les 16 et 17 février 2011 ${ }^{(1)}$.

\section{Emergence d'une communauté de recherche}

(1) Le colloque a été organisé par les laboratoires Triangle (UMR 5206) et Lefi (EA 4012), appuyés par l'université LumièreLyon 2, sa chaire d'entrepreneuriat en économie sociale et solidaire, I'Institut des sciences de I'homme (ISH) et I'ENS Lyon, où il s'est tenu. II a été soutenu par I'Institut Veblen pour les réformes économiques, la Fondation Charles Léopold Mayer pour le progrès de l'homme (FPH), I'Institut Caisse des dépôts et consignations pour la recherche, le Cluster 12 "Dynamiques sociales et territoriales" de la région Rhône-Alpes, la région Rhône-Alpes, la Direction générale à la langue française et aux langues de France du ministère de la Culture et de la Communication la coopérative RES et le conseil général du Rhône.
Lappel à communications cherchait à faire émerger des bilans de la dynamique enclenchée il y a bientôt trois décennies, sous forme d'études monographiques comme d'analyses comparatives sur le plan international ou historique, mais il encourageait aussi les approches théoriques ${ }^{(2)}$. Le colloque a accueilli cinquante-trois communications, pour la plupart de chercheurs étrangers. Il a été doublé d'une journée destinée aux acteurs praticiens, élus locaux ou chargés de mission. Au total, 264 personnes, venant de plus de vingt pays, ont participé à tout ou partie de ces trois jours.

Le colloque a contribué à faire émerger et à identifier une communauté de recherche jusqu'ici éparpillée et peu consciente d'elle-même. Il a permis de souligner la faiblesse de l'infrastructure intellectuelle à même de rassembler les chercheurs, mais aussi son émergence progressive: apparition de sites dédiés aux ressources relatives aux monnaies sociales et complémentaires ${ }^{(3)}$, dont un site bibliographique de recension des écrits ${ }^{(4)}$, et de l'International Journal of Community Currency Research (IJCCR) ${ }^{(5)}$, la seule revue consacrée à ces questions.

\section{Des dispositifs très divers}

(2) Appel et communications consultables sur http://triangle. ens-lyon.fr/spip.php?article1588. (3) www.complementarycurrency. org.

(4) www.cc-literature.org.

(5) www.ijccr.net.
Bien que les quatre textes présentés dans ce dossier ne prétendent pas fournir une vue représentative du colloque, ils illustrent la diversité des expériences et des angles de vue. Ils montrent d'abord leur dispersion géographique: sont abordées les expériences en France, via les systèmes d'échange local et la monnaie SOL à Grenoble, en Amérique du Nord, via des réseaux d'échange de proximité québécois, et en Amérique du Sud, via la Banco Palmas au Brésil. Les dispositifs analysés permettent d’aborder 
(6) Blanc J., 2011, "Classifying "CCs": Community, complementary and local currencies' types and generations", International Journal of Community Currency Research, vol. 15, "Special issue on complementary currencies: State of the art ", p. 4-10. les quatre générations qui ont émergé depuis les années 80 dans le monde ${ }^{(6)}$. Le cas des systèmes d'échange local (SEL) français, étudiés par Cyrille Ferraton et David Vallat, et de la plupart des réseaux d'échanges de proximité québécois, étudiés par Manon Boulianne et Yvan Comeau, constitue une première génération: ce sont des monnaies de crédit mutuel, inconvertibles, intégrant l'échange de biens comme de services; leur vocation est davantage sociale qu'économique.

L'Accorderie québécoise, étudiée par Marie Fare, constitue une seconde génération de dispositifs, qui dérive de la première: le crédit mutuel est basé sur le temps, les échanges sont centrés sur des services strictement comptabilisés en temps et leur vocation est essentiellement sociale.

Le palmas brésilien, étudié par Genauto Carvalho de França Filho, Ariádne Scalfoni Rigo et Jeová Torres Silva Junior, constitue sans doute le cas de succès le plus éclatant de la troisième génération de dispositifs: le palmas est convertible en monnaie nationale (mais suivant des conditions plus ou moins strictes) et il est employé dans les usages commerciaux courants; sa vocation est d'abord économique.

Enfin, le SOL français, étudié par Marie Fare en regard de l'Accorderie, renvoie à une quatrième génération: c'est une monnaie réunissant plusieurs projets simultanés. Le SOL alpin, qui fait plus particulièrement l'objet de l'analyse, sest en réalité concentré sur la vocation économique d'une monnaie fidélisant des consommateurs dans un réseau de prestataires professionnels agréés, rabattant le projet sur celui d'une troisième génération.

\section{Difficultés et réussites}

Les SEL français sont marqués par le refoulement de transactions marchandes et professionnelles. Cyrille Ferraton et David Vallat les analysent au travers du prisme de la dette et de la réciprocité, à partir d'un cadre analytique empruntant à l'anthropologie. Lutopie d'échanges égalitaires, horizontaux et personnalisés, mais dégagés du marché (dont l'idéal promeut pourtant l'horizontalité et l'égalité formelle des agents) comme des liens hiérarchiques (qui construisent pourtant un échange personnalisé), ne prend vie que par la construction de dispositifs de sensibilisation. Cet équilibre fragile menace toujours de basculer soit dans une solidarité charitable ou philanthropique, soit dans la reproduction des logiques marchandes externes. Le texte de Manon Boulianne et Yvan Comeau éclaire le cas des réseaux d'échange de proximité québécois via une enquête quantitative menée auprès d'adhérents de douze dispositifs de première et de seconde génération. L'étude des motifs d'adhésion et des retombées perçues par les adhérents interrogés montre une combinaison de motifs idéologiques, environnementaux et sociaux et de motifs économiques. Ces derniers apparaissent cependant en second plan, à cela près que les adhérents à bas revenus y sont davantage sensibles. Pour beaucoup des participants interrogés, la cessation de fonctionnement du réseau ne détériorerait que très modérément leur 
situation économique: cela illustre, à microéchelle, la faiblesse économique de ces dispositifs, qu'il faut cependant nuancer en fonction du niveau de revenu des participants.

Le texte de Marie Fare établit un pont entre deux rivages de l'Atlantique et entre deux générations de dispositifs: l'Accorderie de Québec et le SOL de Grenoble, étudiés par enquêtes quantitative et qualitative. Elle les interroge sous l'angle d'un développement local soutenable, évalué par trois critères: la territorialisation des activités, la dynamisation des échanges et la transformation des pratiques, des modes de vie et des représentations sociales. Si l'Accorderie, qui a réussi à créer un sentiment de communauté locale solidaire, est un succès dans ces trois critères, il n'en est pas de même du SOL alpin. Confiné au volet marchand du projet SOL et pénalisé par sa petite taille, son impact le plus significatif semble être celui de la transformation des représentations sociales dans le sens d'un développement local soutenable. Retraversant l'Atlantique, Genauto Carvalho de França Filho, Ariádne Scalfoni Rigo et Jeová Torres Silva Junior analysent le cas emblématique de la Banque Palmas, dans un quartier pauvre et excentré de Fortaleza, au Brésil. Ils soulignent le lien fort entre la finance solidaire (via le microcrédit) et la monnaie sociale, via une forme d'autogestion communautaire qui est à l'origine de cette «banque communautaire de développement » de forme associative. Après une première expérience frustrante de dispositif de première génération (le palmares), la banque communautaire a modifié le projet, qui est devenu de troisième génération avec le palmas, une monnaie convertible à usage commercial. Le bilan de dix années de Banco Palmas montre que la réussite du dispositif en matière de lutte contre la pauvreté tient à la combinaison de la finance solidaire et de la monnaie sociale dans un cadre autogéré appuyé par une reconnaissance institutionnelle.

\section{Questions transversales}

La succession de ces quatre textes fait apparaitre des questions transversales. Sur la nature de ces projets: sont-ils d'abord symboliques, sociaux, économiques, environnementaux? Si la réponse se trouve dans la combinaison de ces critères, quelle doit être leur articulation propre à chaque génération de systèmes? Ensuite, ces textes posent la question de la pérennité de ces dispositifs: sont-ils destinés à durer - mais alors, tant il semble que la pérennité suppose un appui financier extérieur durable, à quelles conditions? - ou sont-ils simplement des outils de transition destinés à disparaitre une fois leur objectif réalisé? Enfin, et en conséquence, quelles conditions doivent être satisfaites pour que ces projets aient un impact significatif? Les articles de ce dossier conduisent à souligner au moins les éléments suivants: un pilotage fin équilibrant les tensions internes entre projets politiques divergents, un nombre suffisant d'utilisateurs et une diversité importante des biens et services proposés. Tel est sans doute l'enjeu majeur que doivent affronter les concepteurs de monnaies sociales et complémentaires dans les années à venir. 